\title{
Vitelliform macular lesions
}

\author{
HAROLD W. SKALKA \\ From the Combined Program in Ophthalmology, University of Alabama in Birmingham- \\ Eye Foundation Hospital, Birmingham, Alabama
}

SUMMARY Two patients with vitelliform macular lesions, normal EOG Arden ratios, and no family history of Best's vitelliform dystrophy are presented. Their findings are compared with those in 10 similar reported cases, and a common, nongenetic aetiology is suggested for all. The several names given to this identical fundus picture by different authors are examined and are seen to be inappropriate. A common, descriptive terminology for all such lesions is suggested.

Until recently the vitelliform phase of Best's macular dystrophy was considered to present both a pathognomonic fundus picture and a pathognomonic electrophysiological profile consisting of a normal electroretinogram (ERG) and an abnormal electrooculogram (EOG).

In 1973 Birndorf and Dawson ${ }^{1}$ described a patient with a 'typical sunny-side-up egg yolk lesion of each macula' whom they diagnosed as having Best's vitelliform dystrophy, but who had clearly normal EOG ratios. In 1977 Kingham and Lochen ${ }^{2}$ described 6 patients with vitelliform macular lesions and normal (in 11 of 12 eyes) EOG ratios. As in the case of Birndorf and Dawson these patients had no family history of ocular disease (vitelliform dystrophy is inherited as an autosomal dominant). As was also true of the previously described case, Kingham and Lochen's patients were older than the usual Best's patient with an intact vitelliform lesion. Kingham and Lochen considered these patients to be distinct from patients with Best's vitelliform dystrophy and termed the fundus picture they observed 'vitelliform macular degeneration'.

In the same year Fishman and coworkers ${ }^{3}$ described 3 patients with morphologically similar fundus lesions and normal EOG ratios. As opposed to the above-mentioned patients, Fishman et al.'s 3 patients showed perifoveal capillary leakage on fluorescein angiography, and their disease was called 'pseudovitelliform macular degeneration'. Although 2 of these patients were related, all 3 were again noted to be older than the typical vitelliform-stage Best's patient.

In the present paper 2 additional patients are

Correspondence to Harold W. Skalka, MD, 1720 Eighth Avenue South, Birmingham, Alabama 35233, USA. described with vitelliform macular lesions and normal EOG ratios, and some thoughts are presented on terminology and a possible aetiology for these conditions.

\section{Case reports}

CASE 1

A 46-year-old Negro male was found to have $20 / 40$ acuity OD, $20 / 60$ OS (OD $-0.50=+1.00 \times 180$, OS $-0.75=+0.50 \times 180$ ). External and slit-lamp examination was unremarkable. A smooth, round vitelliform lesion was present in the right macula, and marked changes in the retinal pigment epithelium were seen in the left macula (Fig. 1). Scattered chorioretinal lesions were present bilaterally, especially along the course of the retinal veins. Perivenous sheathing was evident overlying the chorioretinal scars. A diagnosis of Best's vitelliform dystrophy was made, and a retinal consultant concurred in the diagnosis. A fluorescent treponeme antibody absorption test (FTA-abs) was nonreactive, and the patient was referred to us for further testing.

Arden contrast sensitivity test scores $^{4}$ were 101.5 OD, 109 OS (both significantly elevated). An ERG (Cambridge ERG system with Burian-Allen electrodes) was unremarkable except for borderline low-normal photopic voltages and slightly decreased scotopic voltages (a-wave 90 millivolts $\mathrm{OU}, \mathrm{b}$-wave 135 millivolts OD, 165 millivolts OS). An EOG (GT Instrument Co. Model 701 Stimulator, Gould Brush 220 dual channel recorder) yielded Arden ratios of $2 \cdot 14 \mathrm{OD}, 2 \cdot 23 \mathrm{OS}$. Fluorescein angiography OD (Fig. 2) revealed blockage of choroidal fluorescence by the vitelliform macular lesion, with late staining, as well as window defects in the areas of chorioretinal scarring. 


\section{CASE 2}

A 67-year-old emmetropic Negro male with visual acuity of 20/100 OD, 20/80 OS was referred for an EOG to confirm the clinical diagnosis of Best's vitelliform dystrophy. There was no family history of ocular disease.

Ocular examination was remarkable only for the presence of smooth, round, slightly elevated vitelliform lesions in both maculas (Fig. 3). An EOG was performed (instrumentation as in case 1), and yielded Arden ratios of 2.24 OD, 2.09 OS. An FTA-abs was drawn, and was found to be reactive.

Fluorescein angiography OS (Figs. 4 and 5) revealed blockage of choroidal fluorescence by the vitelliform lesion, with late staining seen $\mathrm{OU}$.
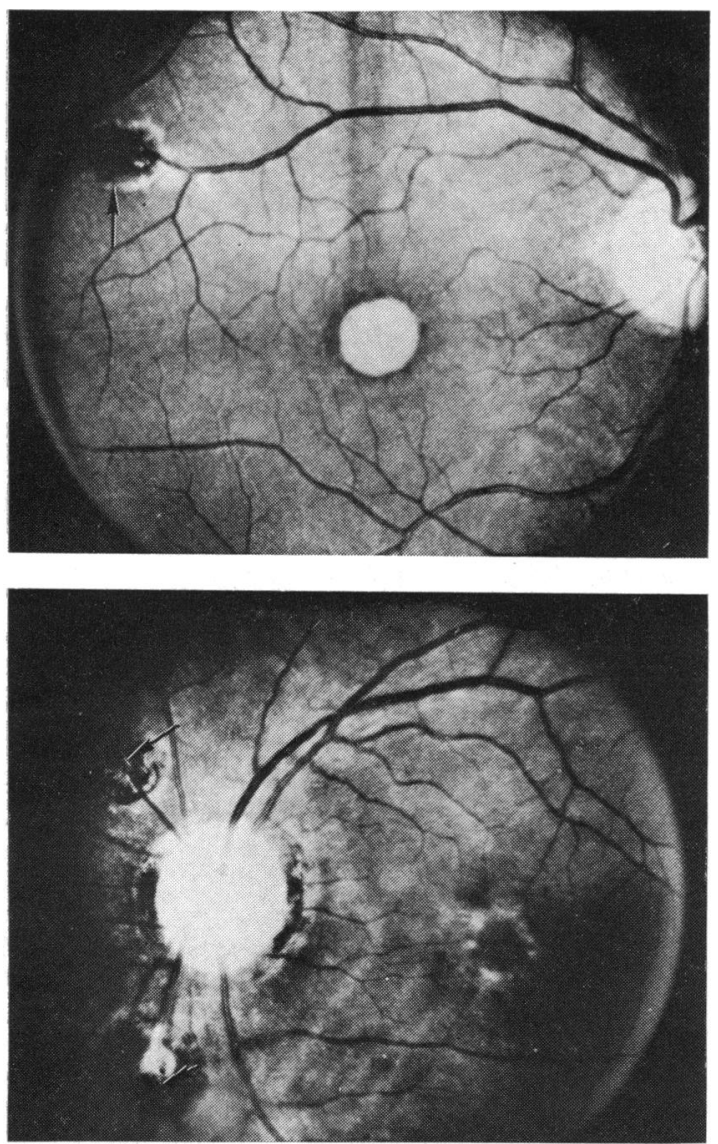

Fig. 1 Case 1. Top: macular area OD. Yellow, vitelliform macular lesion present, as well as chorioretinal scar (arrow) superotemporal to macula. Bottom: posterior pole OS. Macular pigmentary changes present, as well as peripapillary and scattered chorioretinal scars. Perivenous sheathing (arrows) visible in areas of chorioretinal scarring.

\section{Discussion}

Best's disease is a dominantly inherited condition, whose vitelliform phase usually progresses to chorioretinal scarring and atrophy by the 3rd or 4th decade (although this is variable). The 2 patients we report (like the patients of Birndorf and Dawson, Kingham and Lochen, and Fishman et al.) have no family history of Best's disease and are older than the age range normally associated with the intact vitelliform stage of this disorder.

One of our patients (case 1) had clear evidence of bilateral healed chorioretinal lesions, while the other had a reactive FTA-abs. Kingham and Lochen felt that the lesions in 2 of their 6 patients represented
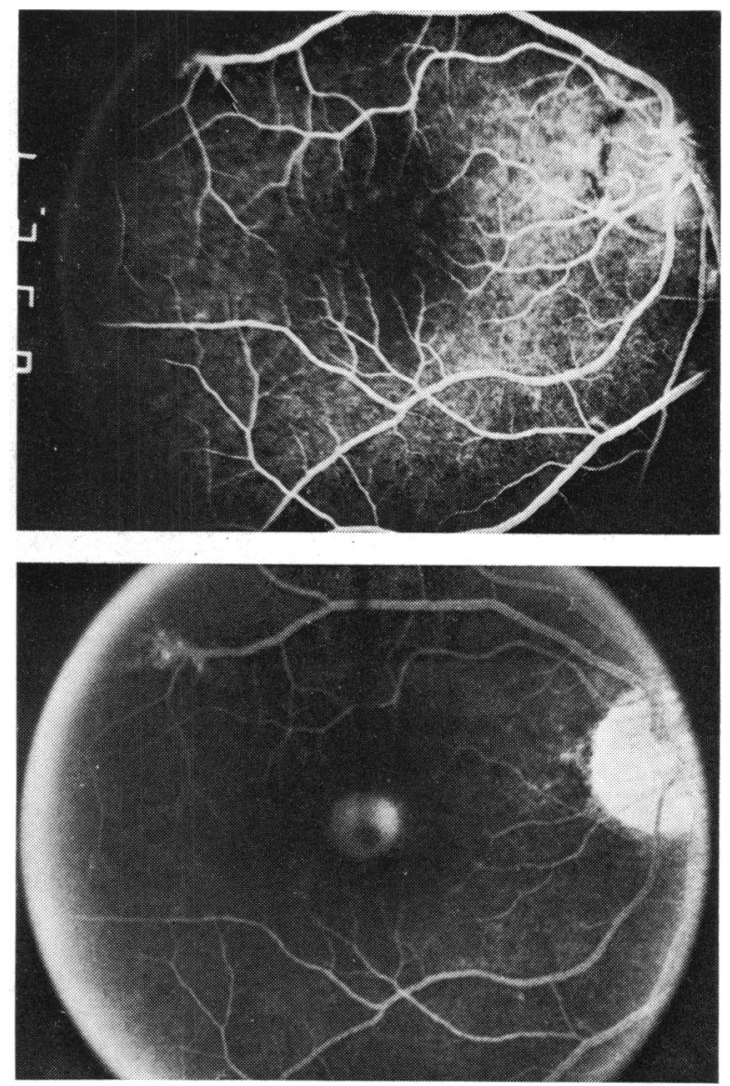

Fig. 2 Case 1. Top: fluorescein angiogram OD, 52.9 seconds after injection. Vitelliform macular lesion blocks underlying fluorescence, with small window defects around its periphery. Window defect seen in area of chorioretinal scarring (arrow). Bottom: fluorescein angiogram OD, 20 minutes after injection. Late staining of vitelliform macular lesion evident. Window defect persists at site of chorioretinal scarring superotemporal to macula. 

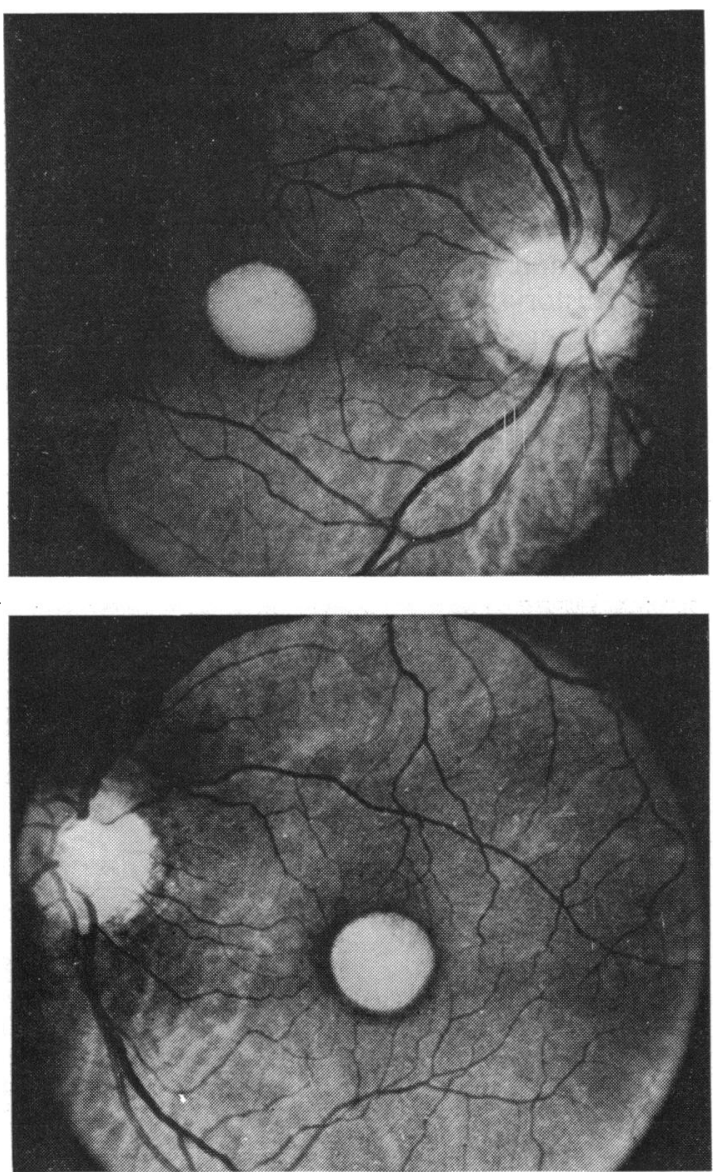

Fig. 3 Case 2. Top: posterior pole OD, showing minimally elevated yellow vitelliform macular lesion. Bottom: posterior pole OS, showing nearly identical vitelliform macular lesion.

a subretinal exudative form of degenerative choroidopathy. Fishman et al.'s 3 patients were all seen in a stage demonstrating active perifoveal capillary leakage.

It is herein proposed that the vitelliform lesions in all these patients might have resulted from fluid leakage into the macular area from inflammation due to various causes (with other visible signs and sequelae, or otherwise), differential reabsorption of the constituents of the leaked fluid, and consequent accumulation (as suggested by Fishman et al. ${ }^{3}$ ) of protein and lipid material in or near the retinal pigment epithelium. Whether or not fluid leakage into the posterior pole could be demonstrated on fluorescein angiography would depend on the stage of the causative process operating at the time of angiography.
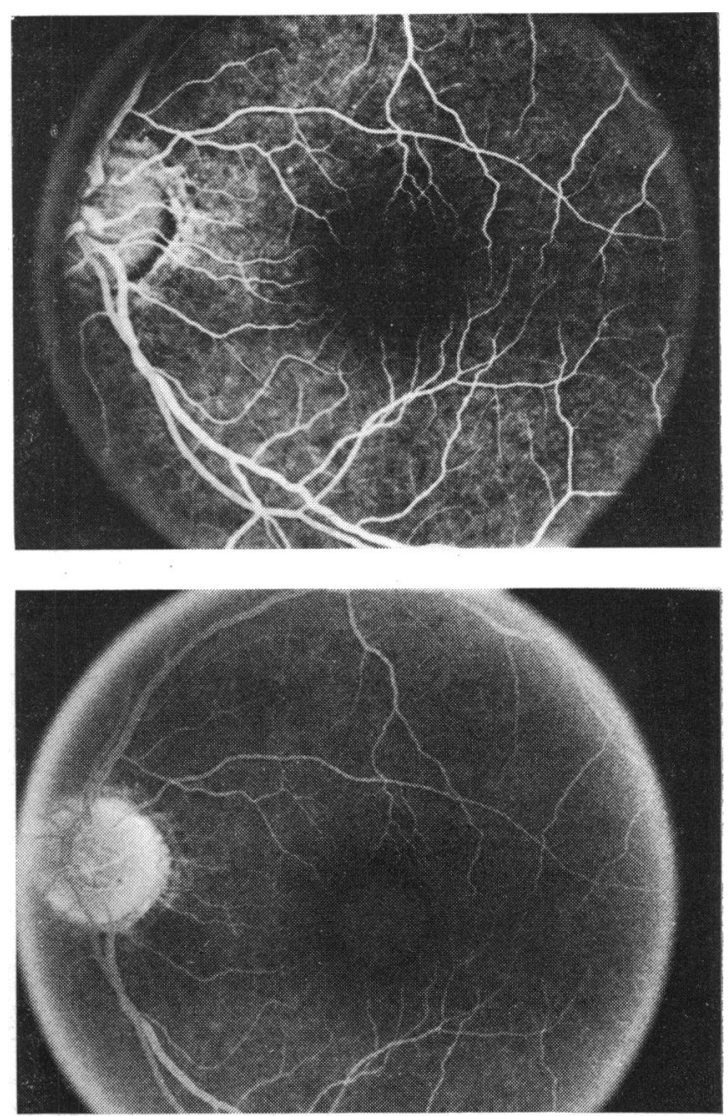

Fig. 4 Case 2. Top: arteriovenous phase fluorescein angiogram OS, $26 \cdot 3$ seconds after injection.

Vitelliform lesion in macula blocks underlying fluorescence. Bottom: fluorescein angiogram OS, 20 minutes after injection. Mild staining of vitelliform lesion can be seen.

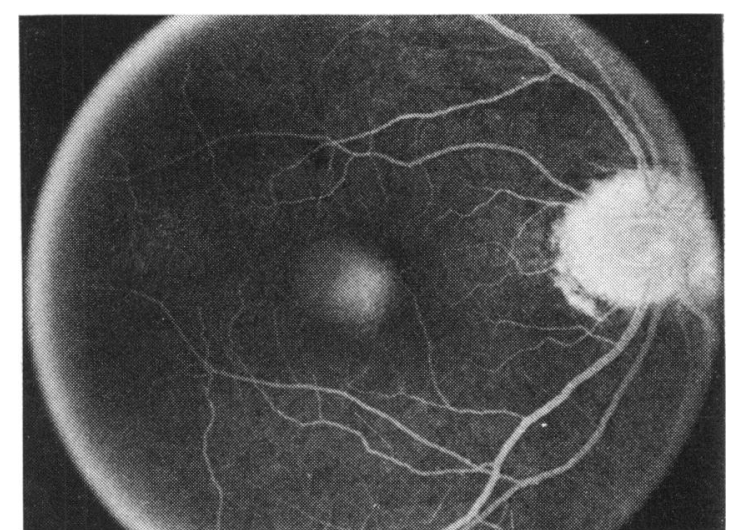

Fig. 5 Case 2, fluorescein angiogram, $O D, 20$ minutes after injection. Staining of vitelliform lesion $O D$ is somewhat more intense than that seen $O S$. 
It is not uncommon in ophthalmology to find different disease processes producing a clinically identical final fundus appearance. The retina is, after all, a small tissue with a limited range of gross pathological alterations available to it. For example, the fundus picture of tapetoretinal dystrophy might be a result of a primary, inherited retinitis pigmentosa variety (whether dominant, recessive, or $\mathrm{X}$-linked) or due to a syndrome of known aetiology with diverse manifestations such as Bassen-Kornzweig syndrome (abetalipoproteinaemia) or Refsum's disease (phytanic acid $\alpha$-hydroxylase deficiency).

The actual pathophysiology operative in these patients with vitelliform lesions, and the extent to which their disease processes are truly related, will of course remain speculative until adequate material can be studied histopathologically. At present there appear to be no such reports concerning eyes with lesions simulating the vitelliform stage of Best's disease.

These patients do not appear to have a genetic dystrophy, and the term 'vitelliform dystrophy' appears to be inappropriate. 'Vitelliform degeneration $^{2}{ }^{3}$ does not appear to be justified, as the underlying cause remains unknown and progression has not been demonstarted as a regular feature in these patients. Since these lesions are truly vitelliform, the 'pseudovitelliform' designation of Fishman et $\mathrm{al}^{3}{ }^{3}$ merely complicates matters. It is therefore suggested that the term 'vitelliform lesion' (which lesion may or may not occur in conjunction with the other findings which accompany Best's dystrophy, such as an abnormal EOG and an appropriate family history) be used as a purely descriptive title for this fundus picture, a picture which can apparently result from multiple causes. The designation 'vitelliform macular dystrophy', along with its eponymic designation 'Best's disease', may be reserved for the genetic entity which so often produces lesions clinically indistinguishable in appearance from those found in the patients discussed here.

\section{References}

1 Birndorf LA, Dawson WW. A normal electro-oculogram in a patient with a typical vitelliform macular lesion. Invest Ophthalmol Visual Sci 1973; 12: 830-3.

2 Kingham JD, Lochen GP. Vitelliform macular degeneration. Am J Ophthalmol 1977; 84: 526-31.

3 Fishman GA, Trimbel S, Rabb MF, Fishman M. Pseudovitelliform macular degeneration. Arch Ophthalmol 1977; 95: 73-6.

4 Arden GB. The importance of measuring contrast sensitivity in cases of visual disturbances. $\mathrm{Br} J$ Ophthalmol 1978; 62: 198-208. 